Proc. of the 15th Int. Workshop on Slow Positron Beam Techniques and Applications, Prague, September 2-6, 2019

\title{
Defects in Thin Layers of High Entropy Alloy HfNbTaTiZr
}

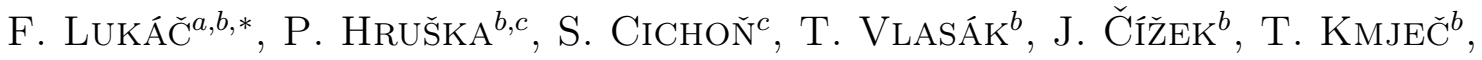 \\ O. Melikhova ${ }^{b}$, M. Butterling ${ }^{d}$, M.O. Liedke ${ }^{d}$ And A. WaGneR ${ }^{d}$ \\ ${ }^{a}$ Institute of Plasma Physics of the Czech Academy of Sciences, Za Slovankou 3, 18200 Prague 8, Czech Republic \\ ${ }^{b}$ Charles University, Faculty of Mathematics and Physics, V Holešovičkách 2, 18000, Praha 8, Czech Republic \\ ${ }^{c}$ Institute of Physics of the Czech Academy of Sciences, Na Slovance 2, 18221 Prague 8, Czech Republic \\ ${ }^{d}$ Institute of Radiation Physics, Helmholtz-Zentrum Dresden-Rossendorf, \\ Bautzner Landstr. 400, 01328, Dresden, Germany
}

\begin{abstract}
High entropy alloys represent a new type of materials with unique combination of physical properties originating due to occurrence of single phase solid solution of numerous elements. Preparation of high entropy alloys films with nanosized grains promises increased effective surface and high intergranular diffusion of elements. In the present work HfNbTaTiZr films were deposited by magnetron sputtering from single phase HfNbTaTiZr target prepared by spark plasma sintering. Chemical composition of high entropy alloys thin films prepared this way was enriched in $\mathrm{Ti}$ and depleted in $\mathrm{Zr}$ and $\mathrm{Nb}$. Very fine microstructure of the film was documented and defect distribution was found to be non-uniform with depth.
\end{abstract}

DOI: 10.12693/APhysPolA.137.219

PACS/topics: 78.70.Bj, 41.75.Fr, 68.35.Dv

\section{Introduction}

High entropy alloys (HEAs) or complex concentrated alloys or multi-principal element alloys [1] represent a group of materials with combination of interesting properties. While most conventional alloys are based on one principal matrix element with alloying elements in lower concentration, HEAs are mixtures of at least four elements in similar atomic ratio. High configurational entropy of this system causes the formation of single solid solution phase at high temperatures [2] and large number of elements promises combination of interesting phases when annealed [3]. Therefore, new combinations of physical properties are expected, namely mechanical, oxidation, and wear resistance or gas absorption. Moreover, HfNbTaTiZr alloy belongs to a group of refractory materials with a high melting point and high thermal stability [4] with enhanced ductility and strength [5]. Casting of this alloy results in dendritic structure and subsequent homogenization annealing produces large grains and the alloy is susceptible to embrittlement from absorbed atmosphere impurities [6]. Moreover, HEAs are widely studied for interaction with hydrogen [7]. For hydrogen absorption, large surface area together with small grains microstructure is advantageous for enhanced absorption/desorption kinetics due to hydrogen diffusion along grain boundaries and increased capacity due to hydrogen trapping in open volume defects [8]. Series of thin films were fabricated by magnetron sputtering in order to study the small grain structure and associated defects.

*corresponding author; e-mail: lukac@ipp.cas.cz

\section{Experimental}

HEA films were deposited by DC magnetron sputtering $(10 \mathrm{~W}, 250 \mathrm{~V}, 40 \mathrm{~mA})$ in an UHV chamber $\left(p_{0}<10^{-6} \mathrm{~Pa}\right)$ in Ar working atmosphere of $2 \mathrm{~Pa}$. A HfNbTaTiZr target with equiatomic composition prepared by spark plasma sintering from gas atomized powder of HfNbTaTiZr alloy [9] was used. Films with thickness of $1100 \mathrm{~nm}$ were deposited on fused silica substrates $\left(10 \times 10 \times 0.5 \mathrm{~mm}^{3}\right)$ at room temperature. Film thickness was determined by profilometry measurement combined with estimation of the deposition rate of $4 \mathrm{~nm} / \mathrm{min}$. The surface structure was observed by scanning electron microscopy (SEM) using Quanta 200 (FEI, USA).

The positron annihilation lifetime spectroscopy (PALS) measurements were performed at the monoenergetic positron spectroscopy (MePS) beamline [10] at the ELBE (Electron Linac for beams with high Brilliance and low Emittance) facility at the HelmholtzZentrum Dresden-Rossendorf. Positron lifetime spectra were measured using a digital spectrometer with the time resolution of $250 \mathrm{ps}$ (FWHM of resolution function).

$\mathrm{X}$-ray fluorescence (XRF) was used for measurement of overall chemical composition of samples by S2 Puma (Bruker, Germany) and for chemical elements mapping with beam of $300 \mu m$ diameter on Eagle III $\mu$ Probe (Roentgenanalytik Systeme GmbH \& Co. KG, Germany).

\section{Results and discussion}

Figure 1 shows surface of the HfNbTaTiZr film. Fine microstructure is composed of cells with size around $100 \mathrm{~nm}$. Cells are separated by interfaces which are clearly visible in Fig. 1. Maps of chemical elements obtained by XRF are shown in Fig. 2. Sputtered thin film 


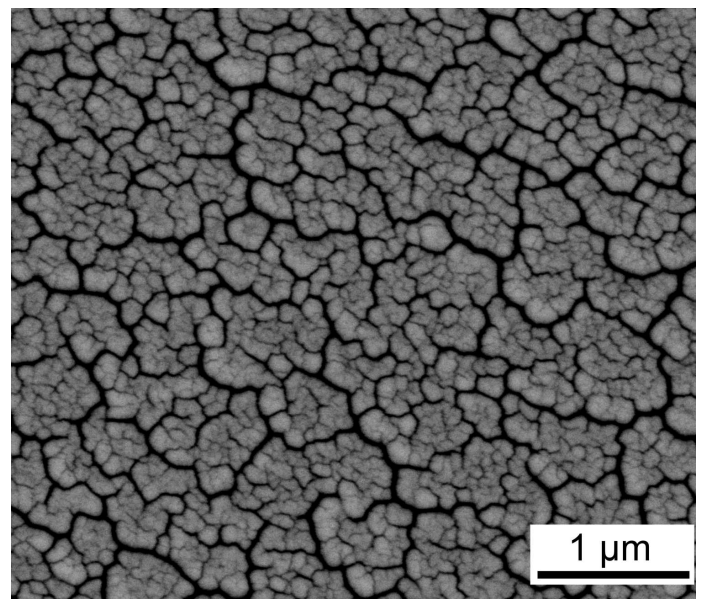

Fig. 1. Surface micrograph of a thin film sputtered from HfNbTaTiZr alloy captured in back scattered electrons mode of SEM.
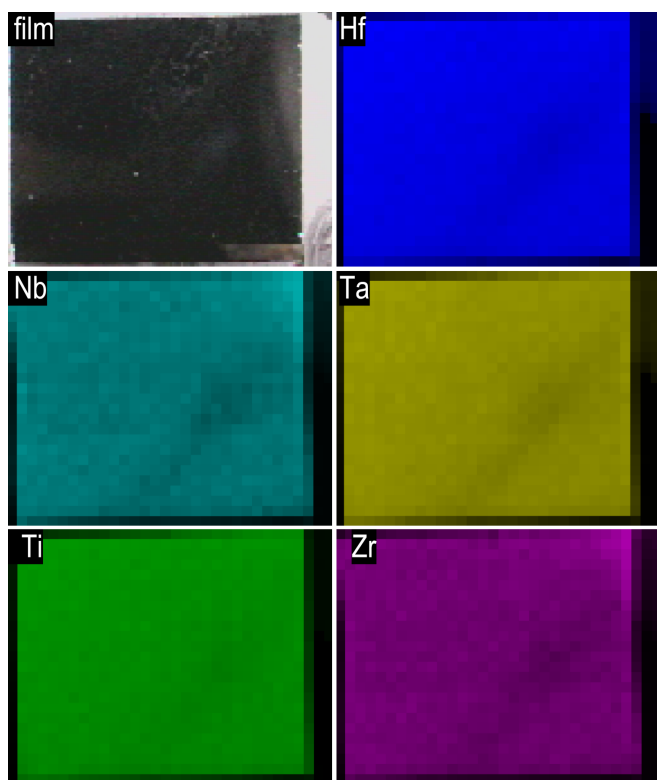

Fig. 2. Maps of chemical elements in the HEA film determined by XRF. The scanned area is $10 \times 10 \mathrm{~mm}^{2}$.

shows no sign of macroscopical chemical heterogeneity. However, chemical composition of sputtered film differs from the composition of sputterring target, see Table I. While the sputtering target prepared by sintering of gas atomized powder has chemical composition close to the equiatomic stoichiometry, sputtered film is enriched in $\mathrm{Ti}$ and depleted by $\mathrm{Nb}$ and $\mathrm{Zr}$. This chemical alteration in the sputtered film can be explained by different sputtering yields of used elements and due to different interaction of each element with argon ions and sputtered atoms in the plasma. The target surface was examined also after magnetron sputtering and was found to be accordingly depleted in $\mathrm{Ti}$ and enriched in $\mathrm{Nb}$ and $\mathrm{Zr}$. This is in accordance with higher sputtering yield of $\mathrm{Ti}$ compared to $\mathrm{Nb}$ and $\mathrm{Zr}$.
TABLE I

Chemical composition in at.\% determined by XRF for a HfNbTaTiZr target before and after magnetron sputtering, and for deposited HEA thin film.

\begin{tabular}{l|c|c|c|c|c}
\hline \hline & $\mathrm{Ti}$ & $\mathrm{Zr}$ & $\mathrm{Nb}$ & $\mathrm{Hf}$ & $\mathrm{Ta}$ \\
\hline target before sputtering & 18.3 & 18.7 & 19.3 & 23.2 & 20.5 \\
target after sputtering & 12.9 & 22.2 & 23.2 & 20.6 & 21.1 \\
thin HEA film & 29.0 & 13.2 & 13.2 & 21.6 & 23.0
\end{tabular}

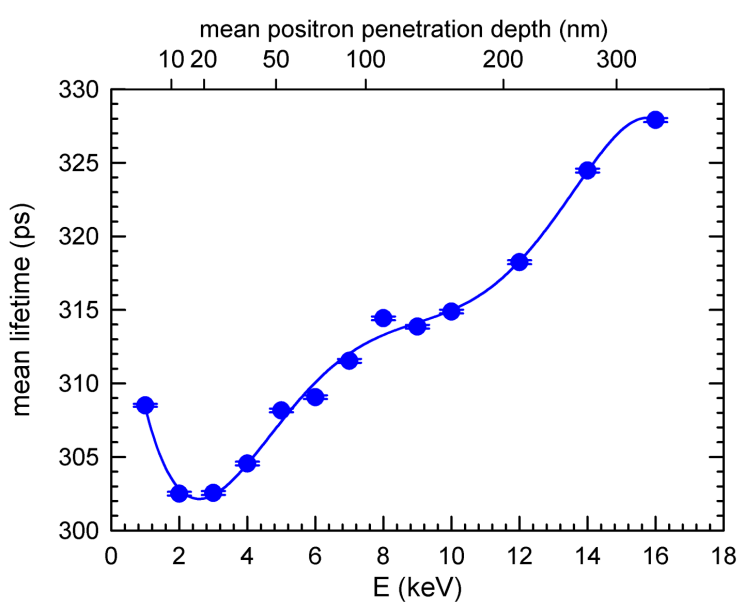

Fig. 3. The dependence of the mean positron lifetime on the energy $E$ of incident positrons. The solid line is just to guide the eyes. The upper horizontal axis shows the mean positron penetration depth.

The HEA film was measured using MePS beam up to positron implantation energy of $16 \mathrm{keV}$ which corresponds to the mean positron penetration depth of $\approx 340 \mathrm{~nm}$ as calculated using the Makhovian implantation profile and assuming the theoretical density of HfNbTaTiZr alloy of $10 \mathrm{~g} / \mathrm{cm}^{3}$. Hence, for a HEA film with thickness of $1000 \mathrm{~nm}$ virtually all positrons annihilate in the film. Figure 3 shows the mean positron lifetime measured in the HEA film plotted as function of the positron energy. The mean positron lifetime exceeds 300 ps and is, therefore, much higher than the bulk lifetime of HfNbTaTiZr alloy which equals 146 ps $[9,11,12]$. This is caused by the fact that due to nanocrystalline cell size the majority of positrons are annihilated at boundaries between cells containing vacancy-like misfit defects and also bigger open volume voids visible in Fig. 1. A high mean lifetime value measured at $1 \mathrm{keV}$ may be associated with formation of Ps at the rough surface of the thin films. The mean positron lifetime first decreases with increase of energy of implanted positrons due to decrease of fraction of positrons diffusing back to the surface, see Fig. 3. However, after reaching a minimum at $3 \mathrm{keV}$ it exhibits a pronounced increase indicating that size or concentration of open volume defects increases with depth. Since it is expected that nanocrystalline size of cells leads to saturated positron trapping at interfaces it is most probable that size of large voids increases with 
depth. This can be connected with penetration of oxygen into the film. Subsurface layers are oxidized first and filling of intercell voids and pores with oxygen decreases their open volume and consequently reduces the lifetime of trapped positrons. At higher depths from surface the concentration of oxygen decreases and reduction of open volume of voids by trapped oxygen is lower. This is reflected by increasing positron lifetime with increase of positron energy.

\section{Conclusions}

Thin HEA film prepared by magnetron sputtering exhibits nanocrystalline cell-like microstructure with a high volume fraction ratio of grain boundaries. Chemical composition of the sputtered film differs from the composition of the target used due to different sputtering yield of different elements. The mean positron lifetime measured in the film exceeds 300 ps indicating presence of larger open volume defects (voids) located at interfaces between grains. The open volume of these defects increases with increase of depth from the surface.

\section{Acknowledgments}

This work was supported by the Czech Science Foundation (project 17-17016S). The MePS facility has partly been funded by the Federal Ministry of Education and Research (BMBF) with the grant PosiAnalyse (05K2013). The support of the ELBE team at HZDR is greatly acknowledged.

\section{References}

[1] D. Miracle, O. Senkov, Acta Mater. 122, 448 (2017).

[2] O. Senkov, J. Scott, S. Senkova, D. Miracle, C. Woodward, J. Alloys Comp. 509, 6043 (2011).

[3] B. Cantor, Entropy 16, 4749 (2014).

[4] O.N. Senkov, J.M. Scott, S.V. Senkova, F. Meisenkothen, D.B. Miracle, C.F. Woodward, J. Mater. Sci. 47, 4062 (2012).

[5] J. Zýka, J. Málek, Z. Pala, I. Andršová, J. Veselý, in: Proc. METAL 2015, 24th Int. Conf. on Metallurgy and Materials, Tanger Ltd. Ostrava 2015, p. 1687.

[6] G. Dirras, L. Lilensten, P. Djemia, M. Laurent-Brocq, D. Tingaud, J.P. Couzini, L. Perrire, T. Chauveau, I. Guillot, Mater. Sci. Eng. A 654, 30 (2016).

[7] C. Zlotea, M. Sow, G. Ek, J.P. Couzinié, L. Perrière, I. Guillot, J. Bourgon, K. Møller, T. Jensen, E. Akiba, M. Sahlberg, J. Alloys Comp. 775, 667 (2019).

[8] P. Hruška, J. Čížek, J. Knapp, F. Lukáč, O. Melikhova, S. Mašková, L. Havela, J. Drahokoupil, Int. J. Hydrogen Energy 42, 22557 (2017).

[9] F. Lukáč, M. Dudr, R. Mušálek, et al., J. Mater. Res. 33, 32473257 (2018).

[10] A. Wagner, W. Anwand, A.G. Attallah, et al., J. Phys. Conf. Series 791, 012004 (2017).

[11] F. Lukáč, M. Dudr, J. Čížek, et al., Acta Phys. Pol. A 134, 891 (2018).

[12] F. Lukáč, R. Mušálek, M. Vilémová, J. Č́ížek, J. Kuriplach, J. Stráská, J. Zýka, J. Málek, AIP Conf. Proc. 2182, 050013 (2019). 